\title{
Fluispotter, a novel automated and wearable device for accurate volume serial dried blood spot sampling
}

\author{
Khem Bahadur Adhikari ${ }^{1}$ (i) , Morten Rohde 2 , Sten Velschow ${ }^{2}$ (D), Ulla Feldt-Rasmussen ${ }^{3}$ (D), \\ Jesper Johannesen ${ }^{4}$ (i) \& Anders Holten Johnsen*,1 (iD) \\ ${ }^{1}$ Department of Clinical Biochemistry, KB3011, Rigshospitalet, Copenhagen, Denmark \\ ${ }^{2}$ Fluisense Aps, Lillerød, Denmark \\ ${ }^{3}$ Department of Endocrinology \& Metabolism, Rigshospitalet, Copenhagen, Denmark \& Faculty of Health \& Medical Sciences, \\ Copenhagen University, Denmark \\ ${ }^{4}$ Department of Pediatrics, Herlev \& Gentofte Hospital, Copenhagen, Denmark \& Faculty of Health \& Medical Sciences, \\ Copenhagen University, Denmark \\ *Author for correspondence: Tel: +45 35453007; Fax: +45 35454640; anders.holten.johnsen@regionh.dk
}

Aim: A novel automated serial dried blood spot (DBS) sampler, 'Fluispotter', was tested for its sampling performance. Materials \& methods: An LC-MS/MS method was developed for the analysis of cortisol in DBS samples serially spotted by Fluispotter. The cortisol concentrations in 148 paired DBS and plasma samples were compared across a hematocrit (HCT) range of 22-55\%. Results: The interassay accuracy and precision were $<10 \%$. Overall assay bias was negligible across the HCTs tested when analyzing the whole-spot DBS samples. The accuracy and precision of the blood volume in $10 \mu \mathrm{l}$ DBS samples spotted by Fluispotters and micropipettes were within 3\%. Deming regression and Bland-Altman analysis showed a good agreement of DBS-predicted and measured plasma cortisol. Conclusion: The Fluispotter performed serial sampling with high accuracy and precision of the sample blood volume.

\section{Graphical abstract:}

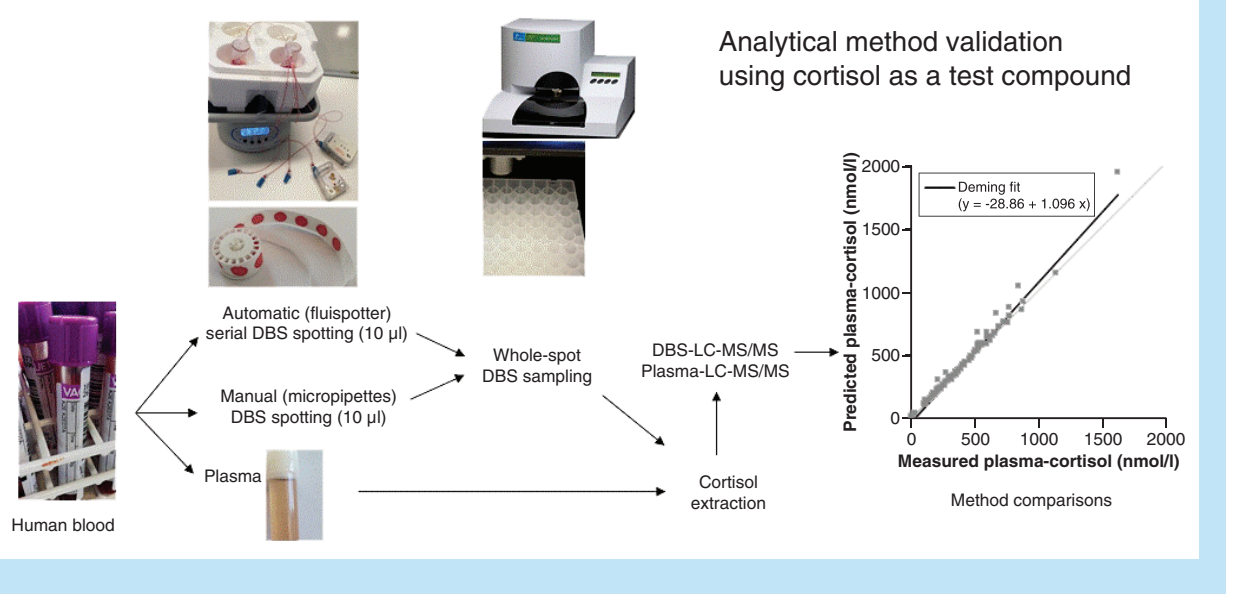

First draft submitted: 26 February 2020; Accepted for publication: 14 May 2020; Published online:

3 June 2020

Keywords: cortisol • dried blood spot • fluispotter • hematocrit $\bullet$ LC-MS/MS • microsampling

The use of dried blood spot (DBS) analysis has increased in various applications [1-3] after the first report by Guthrie and Susi in 1963 for the estimation of phenylalanine in newborns to diagnose phenylketonuria [4]. DBS has many 


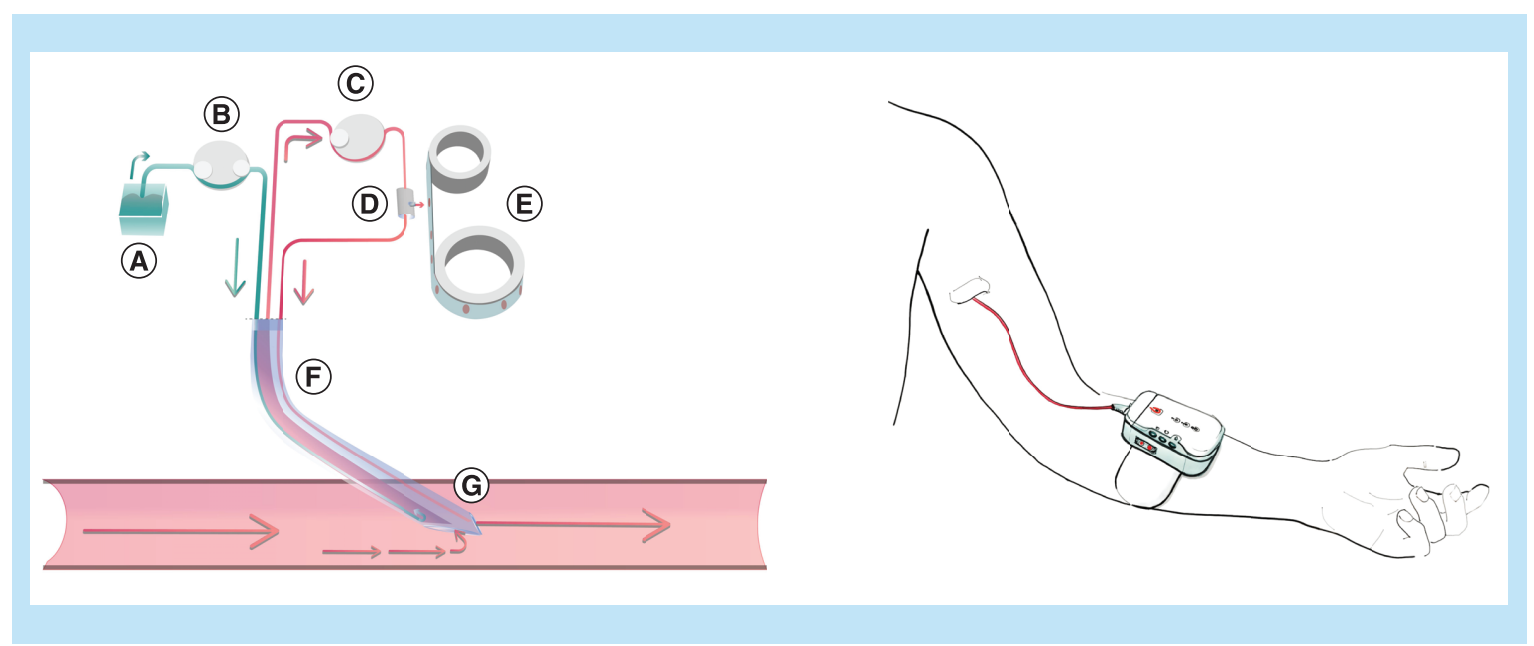

Figure 1. Fluispotter technology. Left side: (A) Reservoir for sodium citrate flushing solution, (B) pump for flushing solution, (C) blood pump, (D) valve for uni-directional flow, (E) PerkinElmer 226 paper strip. A-E are all enclosed in the sterile single-use Fluispotter Cartridge. (F) Multi-lumen catheter with (G) Tip of catheter placed inside a peripheral vein. The catheter tip is designed to mix sodium citrate flushing solution to the blood flowing into the cartridge and to ensure that return-blood is not mixed with the fresh blood sample. Right side: Sketch of Fluispotter placed on the left lower arm, with the sampling catheter placed in the brachial vein in the upper arm.

advantages over conventional whole blood, plasma or serum sampling, especially due to its easy collection, storage and transport [5].

Hematocrit (HCT) can influence the blood spot area, homogeneity and extraction recovery, thereby affecting the overall assay reliability of quantitative bioanalysis of DBS samples [6-8]. There is an increasing interest in accurate volume DBS analysis as it eliminates the HCT-based area (volume) bias. Recently, new blood collection technologies such as volumetric absorptive microsampling $\left(\mathrm{VAMS}^{\mathrm{TM}}\right)$ applied in Mitra ${ }^{\mathrm{TM}}$, microfluidic-based sampling applied in hemaPEN ${ }^{\top \mathrm{M}}$ and $\mathrm{HemaXis}^{\mathrm{TM}}$, and the gravimetric method applied in volumetric DBS card are available for accurate volume blood microsampling [9-15] which has increased the potential of DBS samples in quantitative bioanalysis. Regardless of the sampling method, manual blood sampling triggers a physiological stress response [16] and can be a problem in some patients, in other words, patients with medical conditions leading to bruises, especially upon repeated skin pricks for serial blood sampling $[17,18]$. Hence, monitoring actual concentrations of stress-related hormones such as cortisol can be affected significantly [16,18].

Manual blood spotting is labor intensive, time consuming and may introduce human errors. Automated serial blood sampling devices provide an alternative to manual serial blood sampling. Moreover, unlike in finger prick samples, automated devices can reduce the risk of sampling a mixture of venous and arterial blood plus tissue fluid from the puncture site. A few devices, including Instech ABS2 ${ }^{T M}$ [19] and AccuSampler ${ }^{\circledR}{ }_{[16]}$, have been applied for unattended automated DBS sampling in freely moving animal models, leading to stress-free sampling. However, these devices are not wearable and thus not appropriate for serial DBS sampling in nonhospitalized humans.

Fluispotter ${ }^{\circledR}$ - not yet available for clinical use, is a wearable, programmable and fully automated serial dried blood spot sampling device. Fluispotter is based on patented technology [20-22] consisting of a rechargeable control unit with motors and battery, a sterile single-use cartridge with tubing, reservoir for flushing solution, pumps and filter-paper and a sterile single-use multi-lumen catheter. See technical drawing of Fluispotter in Figure 1 and pictures in Figure 2.

Fluispotter allows for up to $20 \mathrm{~h}$ of unattended serial microsampling of maximum 20 DBS samples of 3 to $10 \mu \mathrm{l}$ venous blood, collected on a strip of PerkinElmer 226 filter-paper at user-defined time points. In the tip of the sampling catheter, which will be placed in the brachial vein in the upper arm, the blood is mixed 9:1 (v/v) with a $4 \%$ sodium citrate flushing solution to prevent coagulation of blood inside the cartridge. The current version of Fluispotter is validated with a $4 \%$ sodium citrate solution but is intended for use with any compatible flushing solution. The control unit logs all information about sampling times and sample volumes. Fluispotter is only to be used by healthcare professionals after appropriate training. 


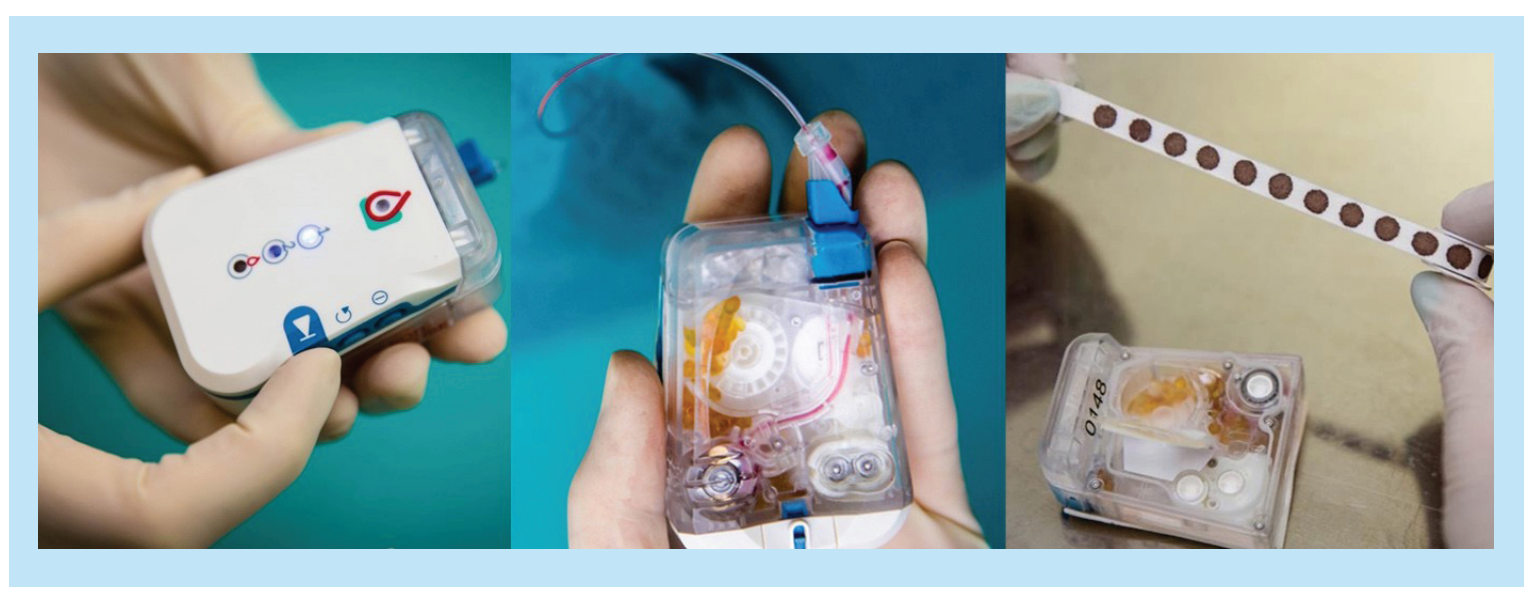

Figure 2. Fluispotter. Assembled device showing top surface (left), bottom surface (middle), and filter paper with dried blood spots removed from the cartridge after sampling completion (right).

Fluispotter could be particularly useful where manual intervention in the sampling period is unwanted, for diagnostic- and treatment-optimizing purposes, in drug monitoring studies as well as in studies of special patient populations including children, for whom low volume blood collection is critically important, for example, for serial biomarker/hormone evaluations.

In this study, Fluispotter was tested for serial DBS microsampling from pooled blood samples in laboratory conditions. The sampling performance of the Fluispotter and the potential of the whole assay to overcome the issues associated with HCT have been investigated by a fully validated DBS-LC-MS/MS quantitative assay using cortisol as a test compound. Data obtained from Fluispotter spotted DBS samples were compared with manually spotted DBS samples from the same pooled blood. Furthermore, cortisol concentrations in paired plasma and manually spotted DBS samples were compared.

\section{Materials \& methods}

\section{Chemicals \& materials}

Cortisol was purchased from Sigma-Aldrich (Broendby, Denmark). Cortisol- $\left[{ }^{13} \mathrm{C}_{3}\right]$ solution was obtained

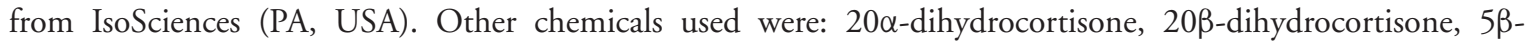
dihydrocortisone, 20(R)-hydroxy prednisolone, and 11-epihydrocortisone, purchased from Toronto Research Chemicals (Toronto, Canada). European Reference Materials ERM ${ }^{\circledR}$-DA192 and -DA193 were supplied by Sigma-Aldrich (Broendby, Denmark). LC-MS grade water and methanol were purchased from Honeywell (Seelze, Germany). Steroid-free serum was obtained from MP Biomedicals (OH, USA).

\section{Preparation of DBS calibrators \& quality control samples}

Two separate primary stock solutions of cortisol (stock A and stock B), one for calibrators and one for quality control (QC) samples, were prepared in methanol, both at a concentration of $0.1 \mathrm{~g} / \mathrm{l}$. Stock solutions of $40 \mu \mathrm{mol} / \mathrm{l}$ cortisol in methanol were prepared and stored at $-20^{\circ} \mathrm{C}$ until use. The difference in LC-MS/MS responses of cortisol in the two stock solutions was $<5 \%$. Working standard solutions of different concentrations were prepared by dilution of the stock solutions with methanol/water, 50/50 (v/v).

DBS calibrators, quality control and test samples for method validation were prepared by spiking suitable concentrations of cortisol solutions in blank (steroid-free) blood. In short, leftover EDTA blood samples (within $48 \mathrm{~h}$ of collection) were obtained from the hospital blood bank. Pooled blood was centrifuged $(1500 \times \mathrm{g}, 10 \mathrm{~min})$, and erythrocytes were harvested and washed thrice with phosphate-buffered saline and combined with steroid-free serum to produce blank blood at three different HCT levels (30, 45 and 60\%).

Working standard solutions of cortisol prepared from stock A at appropriate concentrations were spiked to the blank blood with HCT of $45 \%$ to obtain final concentrations of $0,5,10,20,50,100,300$, and $600 \mathrm{nmol} / \mathrm{l}$ for preparing DBS cortisol calibrators. QC samples at 15, 100 and $400 \mathrm{nmol} / \mathrm{l}$ were similarly prepared by spiking the cortisol working standard solutions from stock B. These pre-spiked calibrators and QC blood samples were further diluted in sodium citrate anticoagulant to mimic the dilution factor $(90 / 10, \mathrm{v} / \mathrm{v})$ of real-time patient sampling by 


\begin{tabular}{|c|c|c|c|}
\hline Time (min) & Eluent A (\%) & Eluent B (\%) & Flow rate $(\mathrm{ml} / \mathrm{min})$ \\
\hline 0.0 & 65 & 35 & 0.400 \\
\hline 0.2 & 65 & 35 & 0.400 \\
\hline 0.25 & 49 & 51 & 0.400 \\
\hline 2.0 & 49 & 51 & 0.400 \\
\hline 2.5 & 1 & 99 & 0.400 \\
\hline 3.3 & 1 & 99 & 0.550 \\
\hline 3.7 & 1 & 99 & 0.550 \\
\hline 3.9 & 65 & 35 & 0.550 \\
\hline 4.5 & 65 & 35 & 0.400 \\
\hline 5.0 & 65 & 35 & 0.400 \\
\hline
\end{tabular}

\begin{tabular}{|c|c|c|c|c|c|}
\hline Substance & Precursor $(m / z)[M+H]^{+}$ & Product $(m / z)[M+H]^{+}$ & Cone (V) & Collision (V) & Dwell time (ms) \\
\hline Cortisol (quantifier) & 363.2 & 121.2 & 20 & 25 & 80 \\
\hline Cortisol (qualifier) & 363.2 & 97.2 & 20 & 25 & 80 \\
\hline Cortisol- $\left[{ }^{13} \mathrm{C}_{3}\right]$ (quantifier) & 366.2 & 124.2 & 20 & 25 & 80 \\
\hline Cortisol- $\left[{ }^{13} C_{3}\right]$ (qualifier) & 366.2 & 100.2 & 20 & 25 & 80 \\
\hline
\end{tabular}

Fluispotter. The spiked blood samples were then mixed for $30 \mathrm{~min}$ at room temperature by gentle shaking in a Mini Shaker (Multi Bio 3D, Biosan, alternating between orbital at $100 \mathrm{rpm}$ for $60 \mathrm{~s}$ and reciprocal at $60^{\circ}$ for 10 s) before spotting by Fluispotter.

Spiked blood for calibrators and QC samples was continuously shaken as described above, while automatically spotting series of DBS samples by five Fluispotter units equipped with PerkinElmer 226 filter paper. Each Fluispotter was programmed to deliver a total of 20 DBS samples of $10 \mu \mathrm{l}$ blood volume each at 3-min intervals. After completion of the sampling procedure, the filter paper with DBS samples was removed from the disposable cartridge and dried overnight at room temperature before storing in zip lock plastic bags at $-20^{\circ} \mathrm{C}$ until analysis.

\section{LC-MS/MS conditions}

Analyses were performed using a Waters ${ }^{T M}$ Acquity ${ }^{T M}$ UPLC system coupled with Waters ${ }^{T M}$ Xevo $^{\circledR}$ TQ-S mass spectrometer, both controlled by MassLynx 4.1 software (Waters ${ }^{T M}$ ). A reverse-phase analytical column (Waters ${ }^{T M}$ Acquity $^{\text {TM }}$ UPLC HSS T3 column $2.1 \mathrm{~mm} \times 100 \mathrm{~mm} \times 1.8 \mu \mathrm{m}, 100 \AA$ ) maintained at $80^{\circ} \mathrm{C}$ was used for chromatographic separation. Mobile phases consisted of water (eluent A) and methanol (eluent B), both with $1.25 \mathrm{mmol} / \mathrm{l}$ ammonium formate. UPLC solvent gradient conditions are shown in Table 1 . The retention time of cortisol was $1.85 \mathrm{~min}$ with a total run time of $5.0 \mathrm{~min}$.

Ionization and MS/MS settings were optimized for best fragmentation and intensity by infusion of the cortisol solution (50/50 methanol/water) in the mobile phase by flow injection analysis. The optimized MS/MS settings were: Capillary $1.5 \mathrm{kV}$, Cone $70 \mathrm{~V}$, desolvation gas $1000 \mathrm{l} / \mathrm{h}$, Cone gas $200 \mathrm{l} / \mathrm{h}$, Nebulizer 7 bar, desolvation temperature $625^{\circ} \mathrm{C}$, source temperature $150^{\circ} \mathrm{C}$. Other operating conditions for MS are shown in Table 2. Electrospray ionization (ESI) was applied in positive mode, and the quantitative and qualitative mass transitions $[\mathrm{M}+\mathrm{H}]^{+}$for both cortisol and cortisol- $\left[{ }^{13} \mathrm{C}_{3}\right]$ internal standard were monitored in multiple reaction monitoring mode.

\section{Method validation}

Method validation experiments were performed to establish the validity of the assay to internationally accepted criteria following the standard guidelines for bioanalytical method validation [23,24]. Special recommendations by The European Bioscience Forum on the validation of bioanalytical methods for DBS were taken into consideration $[25,26]$. 


\section{Cortisol extraction from DBS samples}

Acetonitrile/water: 80/20 (v/v) was used as an extraction solvent of cortisol in DBS samples. In short, the entire (unless stated otherwise) DBS sample was punched into a U-bottom 96-well plate (Thermo Fisher Scientific, AB-0564) using a Wallac DBS Puncher (PerkinElmer, MA, USA). Cortisol was extracted from the DBS with $200 \mu \mathrm{l}$ extraction solvent containing the stable isotopically labeled cortisol- $\left[{ }^{13} \mathrm{C}_{3}\right]$ internal standard $(30 \mathrm{nmol} / \mathrm{l})$ by vigorously mixing on an Eppendorf MixMate (1200 rpm) for $1 \mathrm{~h}$ at room temperature. After centrifugation for $30 \mathrm{~min}$ at $7102 \mathrm{~g}$ (Rotanta 460RF, Hettich), the supernatant was transferred to a 96-well plate and $5 \mu \mathrm{l}$ was then injected for LC-MS/MS analysis.

\section{Blood sampling volume precision}

The reproducibility of the blood sampling volume spotted was evaluated by determining the precision data of the measurements of cortisol in DBS samples $(n=16)$ collected from a pooled blood sample. The reproducibility was tested for DBS samples spotted manually by $10-\mu$ l micropipettes (Drummond Scientific) and automatically by three Fluispotter units.

\section{Precision of in-house DBS-cortisol calibrators}

The reproducibility of the Fluispotter-derived in-house DBS-cortisol calibrators was evaluated by determining the precision data of the measurement of cortisol in DBS samples collected by five Fluispotters, each at different calibrator concentrations $(5-600 \mathrm{nmol} / \mathrm{l})$.

\section{Accuracy \& precision}

The accuracy and precision of the developed method were determined by replicate analyses $(n=6)$ of five levels of quality control DBS samples: LLOQ $(5 \mathrm{nmol} / \mathrm{l})$, low $(15 \mathrm{nmol} / \mathrm{l})$, medium $(100 \mathrm{nmol} / \mathrm{l})$, pooled blood $(\sim 240 \mathrm{nmol} / \mathrm{l}$ ), and high (pooled blood spiked with $200 \mathrm{nmol} / 1$ of cortisol to $\sim 400 \mathrm{nmol} / \mathrm{l})$. The inter-assay accuracy and precision values were derived from three different batches on three different days for low $(15 \mathrm{nmol} / \mathrm{l})$, medium $(100 \mathrm{nmol} / \mathrm{l})$, and pooled blood $(\sim 240 \mathrm{nmol} / \mathrm{l})$ and from two different batches on two different days for LLOQ $(5 \mathrm{nmol} / \mathrm{l})$. The accuracy was determined as percent difference (\% bias) from the nominal concentrations of QC DBS samples, and precision was determined as percent coefficient of variation (\% CV). Acceptance criteria were $\pm 15 \%$ for both accuracy and precision at all concentrations except for the lower limit of quantification (LLOQ), where $\pm 20 \%$ was acceptable.

\section{Recovery}

Recoveries were assessed at three different levels of spiked cortisol concentrations: low $(15 \mathrm{nmol} / \mathrm{l})$ and medium $(100 \mathrm{nmol} / \mathrm{l})$ spiked to blank blood and medium high $(200 \mathrm{nmol} / \mathrm{l})$ spiked to pooled blood. Recovery analyses were performed by replicate analyses $(n=6)$ of spiked quality control DBS samples in three independent assays. The recovery was assessed as follows:

$$
\% \text { Recovery }=\frac{\text { Concentration of the sample with spike }- \text { Concentration of the sample }}{\text { Theoretical concentration of the spike }} \times 100 \% \text {. }
$$

\section{Matrix effect}

Matrix interference was assessed following the method mentioned in previous studies [8,27]. DBS matrix interference on the LC-MS/MS detector response was monitored quantitatively by comparing the post-extraction spiked cortisol response (50 and $200 \mathrm{nmol} / \mathrm{l})$ and/or cortisol internal standard $\left(2,3,4{ }^{13} \mathrm{C}_{3}\right.$-cortisol) $(50 \mathrm{nmol} / \mathrm{l})$ in DBS sample matrix versus the response of the same concentration of cortisol and/or cortisol internal standard $\left(2,3,4{ }^{13} \mathrm{C}_{3}\right.$ cortisol) in neat solution (methanol/water, 50:50, v/v). The matrix effect was calculated as the ratio of the peak area of the analyte spiked into the DBS sample matrix after extraction to the peak area of the same amount of analyte in solvent, multiplied by 100 .

$$
\% \text { Matrix Effect }=\frac{\text { Response at post extraction matrix spike sample }}{\text { Response at neat solution }} \times 100 \%
$$


The matrix interference was also expressed as percent suppression determined by comparing the LC-MS/MS cortisol peak area responses for 'post-extraction matrix spike sample' and 'neat solution'.

$$
\% \text { Suppression }=\frac{(\text { Response at neat solution })-(\text { Response at post extraction matrix spike sample })}{\text { Response at neat solution }} \times 100 \%
$$

Qualitative monitoring of the matrix effect was also performed by post-column infusion method. Since cortisol was already indigenously present in DBS samples, cortisol internal standard $\left(2,3,4{ }^{13} \mathrm{C}_{3}\right.$-cortisol $)$ solution $(10 \mathrm{nmol} / \mathrm{l})$ was continuously infused post-column at a flow rate of $40 \mu \mathrm{l} / \mathrm{min}$ and mixed with the column effluent before entering the ionization interface. DBS samples processed according to the standard sample preparation procedure and blank solvents were injected under described chromatographic and spectroscopic conditions. Mass transition and intensity were recorded to analyze a potential influence from the eluting matrix components at the time point of the elution of cortisol.

\section{Carry-over}

Carry-over was assessed by repeated analysis of Fluispotter-generated low $(5 \mathrm{nmol} / \mathrm{l})$ QC DBS samples followed by repeated analysis of high $(600 \mathrm{nmol} / \mathrm{l})$ QC DBS samples and again followed by repeated analysis of the same low $(5 \mathrm{nmol} / \mathrm{l})$ QC sample. It was also evaluated by analysis of a DBS sample at the upper limit of quantification followed by a solvent blank.

\section{Hematocrit effect}

Human blood with HCT values of 30, 45 and 60\% were prepared by mixing an appropriate volume of washed erythrocytes into steroid-free serum. The cortisol solution was spiked to all three HCT QC samples (30, 45 and $60 \%$ ), leading to a final cortisol concentration of $100 \mathrm{nmol} / \mathrm{l}$.

The HCT-based area bias was visually qualitatively observed in terms of the spread or size of the blood spot on the filter paper and observing the leftover area of the DBS after performing $4.7 \mathrm{~mm}$ sub-spot punch. The HCT QC samples were analyzed along with the DBS calibration standards prepared to the HCT value of $45 \%$. Recoveries at each HCT level $(30,45$ and 60\%) were determined and the HCT-based recovery bias was calculated comparing with the recovery result at the mean HCT level (45\%).

$$
\% \text { Recovery Bias }=\frac{(\text { Recovery })-(\text { Recovery @45\% HCT })}{\text { Recovery } @ 45 \% \text { HCT }} \times 100 \%
$$

Suppression of the LC-MS/MS peak area responses at each HCT level (30, 45 and 60\%) were determined and the HCT-based suppression bias was calculated comparing with the suppression result at the mean HCT level (45\%).

$$
\% \text { Suppression Bias }=\frac{(100-\% \text { Suppression })}{100-\% \text { Suppression @ 45\% HCT }} \times 100 \%
$$

Finally, HCT-based overall assay bias at varying HCT levels (30, 45 and 60\%) was assessed by comparing the cortisol concentration at a specific HCT level with the cortisol concentration at the average HCT level (45\%). A bias within $\pm 15 \%$ of the nominal value would suggest a negligible HCT effect in the given range (30-60\%).

$$
\% \text { Overall Assay Bias }=\frac{(\text { Analyte concentration })-(\text { Analyte concentration @ } 45 \% \mathrm{HCT})}{\text { Analyte concentration @ } 45 \% \mathrm{HCT}} \times 100 \%
$$

\section{Calibration \& linearity}

The linearity of the analytical method was evaluated by analyzing cortisol in DBS calibration samples on eight separate days. Seven levels of DBS calibration samples covering the expected range of 5-600 nmol/l were processed with an internal standard. A DBS calibration curve of the peak area ratio of cortisol with its internal standard (response) was plotted against the nominal concentration of cortisol $(5-600 \mathrm{nmol} / \mathrm{l})$. Concentrations were determined using TargetLynx (Waters ${ }^{\top M}$ ) fitting calibration curve in linear regression with reciprocal fit weighting $(1 / \mathrm{x})$. 


\section{Sensitivity \& measurement range}

Lower limit of detection (LLOD) and lower limit of quantification (LLOQ) were determined as the lowest concentrations at which the signal-to-noise $(\mathrm{S} / \mathrm{N})$ ratios were $\geq 3$ and $\geq 10$, respectively. Two DBS samples of $3500 \mathrm{nmol} / 1$ were extracted and serially diluted (1:1) down to $6 \mathrm{nmol} / 1$ with blank DBS extract and analyzed for the evaluation of dilution integrity.

\section{Selectivity}

The selectivity of the developed method was tested by analyzing blank DBS samples and DBS samples with cortisol at a concentration near the LLOQ, both without and with spiking of possible interfering endogenous isobaric steroids ( $20 \alpha$-dihydrocortisone, $20 \beta$-dihydrocortisone, $5 \beta$-dihydrocortisone, 11 -epihydrocortisone). The baseline resolution and any effect on peak shape or quantitation of cortisol were observed to ensure that no interfering peaks co-eluted with cortisol.

\section{Stability}

Cortisol stability testing in DBS was carried out at selected storage conditions (room temperature, 4 and $-18^{\circ} \mathrm{C}$ ) at different time points over a period of 6 months at two concentrations of cortisol (low: $12 \mathrm{nmol} / \mathrm{l}$ and high: $174 \mathrm{nmol} / \mathrm{l})$. Their mean percent bias of cortisol in stored DBS samples was calculated accordingly, relative to its initial concentration.

\section{DBS-predicted plasma concentration}

DBS samples from 148 individuals with a wide range of known HCT (22\% to 55\%, Supplementary Figure 1) were analyzed for cortisol concentrations and compared with their parallel cortisol concentrations in plasma (measured plasma cortisol) derived from the aliquot of the same blood. Since the majority of blood cortisol is distributed in plasma [28], variables such as HCT and blood-plasma partitioning were considered for appropriate comparisons. DBS to plasma cortisol ratio was calculated for individual samples and plotted against the patient-specific HCT values. The regression equation of this relationship, the DBS cortisol concentration and the individual HCT factor were used to predict the plasma concentration (DBS-predicted plasma concentration). The relationship and agreement between DBS-predicted plasma cortisol and measured plasma cortisol were evaluated to compare the equivalence of the DBS-predicted plasma cortisol with measured plasma cortisol.

The regression equation from the relationship between DBS/plasma ratio and HCT:

$$
\left(C_{D B S} / C_{\text {plasma }}\right)=a+b H C T
$$

The DBS-predicted plasma cortisol concentration can thus be derived from this regression equation, as follows:

$$
\mathrm{PC}_{\text {plasma }}=\frac{\mathrm{C}_{\text {DBS }}}{(\mathrm{a}+\mathrm{bHCT})}
$$

where $C_{D B S}$ is the measured DBS-cortisol concentration, $C_{\text {plasma }}$ is the measured plasma cortisol concentration, $P C_{\text {plasma }}$ is the DBS-predicted plasma cortisol concentration, $a$ is the intercept, and $b$ is the slope from the regression equation.

\section{Data interpretation \& statistical analysis}

Data acquisition and processing were controlled by MassLynx 4.1 software (Waters ${ }^{\top M}$ ) followed by visual inspection. All the statistical calculations were performed using Microsoft Office Excel ${ }^{\circledR} 2016$ (Microsoft Inc., WA, USA) and add-in Analyse-it statistics software, version 5.10 (Analyse-it Software, Ltd., Leeds, UK). Deming regression analysis was performed to calculate constant and proportional bias between different methods. The $95 \%$ confidence intervals (CIs) of the slope and intercept of the fitted regression models were used to check whether the proportional bias and constant bias were significantly different from 1 and 0 , respectively. The level of significance was set at $5 \%$. To study the level of agreement between methods, Bland-Altman analysis was performed using measured plasma cortisol concentrations as reference standard. 


\section{Results \& discussion}

Method development

Sample preparation

The two extraction solvents, methanol/acetonitrile/acetone: 50/25/25 (v/v/v) and acetonitrile/water: $80 / 20$ $(\mathrm{v} / \mathrm{v})$, did not differ significantly in terms of recovery and variation, while pure acetonitrile was the least effective extraction solvent. The methanol/water mixture: 80/20 (v/v) yielded high baseline noise intensity compared with solvent mixture of acetonitrile/water: 80/20 (v/v). Thus, acetonitrile/water 80/20 (v/v) was chosen for extracting cortisol from DBS samples for the method validation. A similar solvent mixture was also practiced previously in similar studies $[29,30]$.

\section{The LC-MS/MS method}

A cortisol standard solution was used to tune the mass spectrometer to optimize different MS/MS conditions for better response of cortisol. An ESI probe in positive mode was used to develop the assay. The singly charged protonated molecular ion $(\mathrm{M}+\mathrm{H})^{+}$of $\mathrm{m} / z$ ratio of 363.2 with high intensity fragment ions having $\mathrm{m} / z$ ratio of 121.2 (quantitative) and 97.2 (qualitative) were selected to monitor cortisol in the method. Stable isotope labeled cortisol $\left(2,3,4{ }^{13} \mathrm{C}_{3}\right.$-cortisol) was used as the internal standard with parallel mass transitions of $\mathrm{m} / \mathrm{z} 366.2$ and $\mathrm{m} / \mathrm{z}$ 124.2 and 100.2. The chromatographic conditions were optimized to achieve good specificity, ionization and peak shape.

\section{Method validation \\ Blood sampling volume precision}

The reproducibility of the blood sampling volume by Fluispotter was evaluated by precision data of the measurement of cortisol in DBS samples collected by three Fluispotters from a human blood pool. Investigation of serial spotting of $10 \mu \mathrm{l}$ whole blood by the Fluispotters, overnight drying at room temperature and subsequent measurement of cortisol in whole-spot analysis within and between Fluispotters resulted in precision data, \%CV, <3\%.

The bias and repeatability of the blood sampling volume $(10 \mu \mathrm{l})$ spotted automatically by Fluispotter and manually by $10-\mu$ l micropipettes (Drummond Scientific) from the same pool of blood were highly comparable when determining the total cortisol concentration and the precision data of the measurements of cortisol in DBS samples. The mean blood volume calculated from cortisol concentrations in DBS samples spotted by these two methods differed by $3 \%$ with a similar level of precision $(\% \mathrm{CV},<3 \%)$. This variation in blood volume spotting was in agreement with other accurate volume DBS sampling systems [10,14,15]. All these results supported the delivery by Fluispotter of accurate and precise sample blood volumes in the form of DBS.

\section{Precision of in-house DBS-cortisol calibrators}

The repeatability of the Fluispotter-derived in-house DBS-cortisol calibrators was evaluated by determining the precision of the measured cortisol in DBS calibrators spotted by five different Fluispotters, each at seven different calibration points $(5-600 \mathrm{nmol} / \mathrm{l})$. The $\% \mathrm{CV}$ was $<5 \%$ at all calibrator levels $(\mathrm{n}=8)$.

\section{Accuracy \& precision}

The accuracy and precision of the method are shown in Table 3. Overall, both were $<13 \%$ at all concentrations, fulfilling the requirement for proper method validation [23,24]. Precision data were comparable with DBS data generated by another accurate volume DBS sampling system, hemaPEN [11].

\section{Recovery}

Recoveries of DBS cortisol at three different spiked concentrations (15 and $100 \mathrm{nmol} / \mathrm{l}$ spiked in blank blood and $200 \mathrm{nmol} / \mathrm{l}$ in a human blood pool) were assessed in replicate analyses $(\mathrm{n}=6)$ in three independent assay runs ( $\mathrm{n}=18$ total replicates at each DBS QC level). The recoveries at all three concentration levels ranged from 94 to $108 \%$ (Table 4 ) and the precision $(\% \mathrm{CV}$ ) was $<7 \%$ at all analyzed concentrations. Recovery was consistent, reproducible, and within the commonly accepted range at all three concentrations (80-120\%). The recoveries from acetonitrile-based extraction solvent were comparable with recoveries using methanol-based extraction solvent reported in another study [31]. 


\begin{tabular}{|c|c|c|c|c|c|c|c|}
\hline \multirow{2}{*}{\multicolumn{2}{|c|}{ Nominal concentration $(\mathrm{nmol} / \mathrm{l})$}} & \multicolumn{3}{|c|}{ Intra-assay } & \multicolumn{3}{|c|}{ Inter-assay } \\
\hline & & $\begin{array}{l}\text { Mean range } \\
(\mathrm{nmol} / \mathrm{l})\end{array}$ & $\begin{array}{l}\text { Accuracy range } \\
\text { (\% bias) }\end{array}$ & $\begin{array}{l}\text { Precision range } \\
(\% \mathrm{CV})\end{array}$ & Mean (nmol/l) & Accuracy (\% bias) & Precision (\% CV) \\
\hline LLOQ $(5)^{\dagger}$ & & 5.0 to 5.6 & 0.1 to 12.4 & 3.8 to 6.8 & 5.2 & 4.9 & 8 \\
\hline $\operatorname{Low}(15)^{\dagger}$ & & 14.1 to 14.9 & -6 to -0.8 & 1.4 to 6.0 & 14.5 & -3.5 & 2.7 \\
\hline Medium $(100)^{\dagger}$ & & 107.4 to 108.8 & 7.4 to 8.8 & 1.1 to 1.7 & 108.3 & 8.3 & 0.7 \\
\hline Pooled blood & & 221.5 to 244.4 & NA & 0.9 to 3.3 & 232.4 & NA & 3.9 \\
\hline High $(\sim 400)^{\ddagger}$ & $\begin{array}{l}\text { Endogenous } \\
\text { concentration }\end{array}$ & & & & & & \\
\hline High1 & 151.6 & 356.7 & -1.9 & 1.9 & NA & NA & NA \\
\hline High2 & 163.4 & 353.8 & -0.9 & 1.0 & NA & NA & NA \\
\hline High3 & 184.6 & 407.5 & 6.0 & 1.3 & NA & NA & NA \\
\hline \multicolumn{8}{|c|}{$\begin{array}{l}\text { Intra-assay values are given as mean }(\mathrm{n}=6) \text { or mean range of all three independent assay runs for low }(15 \mathrm{nmol} / \mathrm{l}) \text {, medium }(100 \mathrm{nmol} / \mathrm{l}) \text {, and pooled blood, of two independent assa } \\
\text { runs for LLOQ }(5 \mathrm{nmol} / \mathrm{l}) \text { and a single assay run for each of three "High }(\sim 400 \mathrm{nmol} / \mathrm{l}) \text { " levels quality control samples. Inter-assay values were determined from three independent assa } \\
\text { runs on three individual days for low }(15 \mathrm{nmol} / \mathrm{l}) \text {, medium }(100 \mathrm{nmol} / \mathrm{l}) \text { and pooled blood and from two independent assay runs on two individual days for LLOQ ( } 5 \mathrm{nmol} / \mathrm{l}) \text {. Sample } \\
\text { were generated from three different Fluispotters. } \\
\text { † Quality control samples from blank blood spiked with cortisol. }\end{array}$} \\
\hline
\end{tabular}

\begin{tabular}{|c|c|c|c|}
\hline Statistic & Low $^{\dagger}(15 \mathrm{nmol} / \mathrm{l})$ & Medium $^{\dagger}(100 \mathrm{nmol} / \mathrm{I})$ & Medium high $\ddagger(200 \mathrm{nmol} / \mathrm{l})$ \\
\hline Mean recovery (\%) & 93.7 & 108.1 & 103.5 \\
\hline STDEV & 4.8 & 1.7 & 6.8 \\
\hline$\% \mathrm{CV}$ & 5.1 & 1.6 & 6.6 \\
\hline
\end{tabular}

\section{Matrix effect}

The suppression of the response for the post-column infused stable isotope labeled internal standard at the time of cortisol elution $(1.85 \mathrm{~min}$ ) was moderate in comparison to its response in pure solvent (Figure 3). Moreover, post-extraction spiking $(50$ and $200 \mathrm{nmol} / \mathrm{l}$ ) assessment revealed that the average percent matrix effect (\%ME) in comparison to neat solution was 92 and $95 \%$, respectively. This indicated the presence of a minor ion suppression by DBS matrix in comparison to the response in neat solution.

\section{Hematocrit effect}

In this quantitative DBS assay, HCT-based overall assay bias refers to the percentage deviation compared with the cortisol concentration measured in the DBS samples at average HCT level (45\%). Area bias, recovery bias, and suppression bias are three major components contributing to the overall assay bias in quantitative DBS bioanalysis $[6,8]$.

Blood viscosity is HCT dependent, high HCT blood being the most viscous, while the low HCT blood sample spreads onto a wider area of the filter paper than high HCT blood samples, leading to a positive area bias of sub-spot sampling $[6,8]$. A similar effect was also seen in this study when visually observing the leftover area of DBS samples of $10 \mu \mathrm{l} \mathrm{spot} \mathrm{volume} \mathrm{from} \mathrm{varying} \mathrm{HCT} \mathrm{levels} \mathrm{after} \mathrm{sub-spot} \mathrm{sampling} \mathrm{(} 4.7 \mathrm{~mm}$ punch) (Supplementary Figure 2). The area of DBS samples linearly increased with decreasing HCT levels, thereby affecting overall assay bias - especially upon sub-spot analysis [7]. Due to the accurate volume spotting by Fluispotter, there was no issue of HCT-based area bias in this assay when analyzing the entire DBS sample.

Recovery, the other component contributing to overall assay bias, is compound dependent and thus relies on the distribution as well as the extraction efficacy of the analyte from the plasma and red blood cell compartments, which eventually can have different recovery trends for different compound types with varying HCT levels $[6,8]$. Cortisol had no notable recovery bias with varying HCT levels and no noticeable suppression bias with varying HCT levels (Figure 4), as shown for other metabolites in a similar study [8]. Area bias was the single and most 


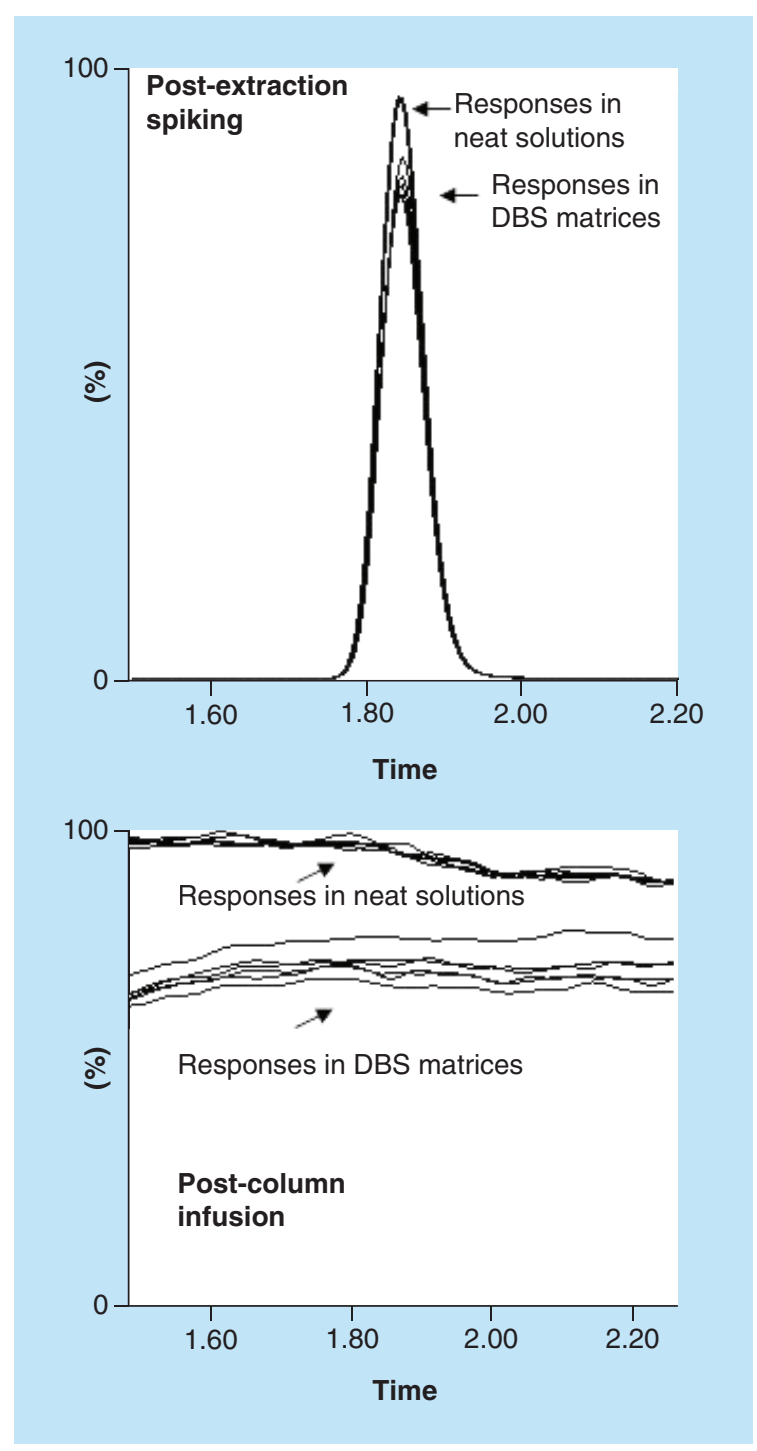

Figure 3. Intensities for the stable isotope labeled Cortisol-[ $\left.{ }^{13} \mathrm{C}_{3}\right]$ internal standard during post-extraction spiking assessment (upper panel) and post-column infusion assessment (lower panel) of matrix effect $(n=6)$.

DBS: Dried blood spot.

influencing component contributing to overall assay bias of almost $\pm 40 \%$ at tested HCT levels of 30 and $60 \%$ relative to the HCT level of $45 \%$ at sub-spot sampling, whereas the effect was negligible at whole-spot sampling (Figure 4). In agreement with cortisol, other compounds such as amlodipine, midazolam, and naproxen analyzed in DBS were also shown to be independent of HCT variations $[2,8]$.

\section{Linearity}

The linearity of the method for DBS cortisol tested by inter-assay analysis of seven DBS cortisol calibrators ranging from 5 to $600 \mathrm{nmol} / \mathrm{l}$ over eight independent assay runs $(\mathrm{n}=8)$ showed a linear correlation $\left(\mathrm{r}^{2}>0.999\right)$ between measured and nominal cortisol concentrations. This was shown by the linear regression equation (average of eight validation runs): measured cortisol $(\mathrm{nmol} / \mathrm{l})=0.507+0.996 \times$ expected cortisol concentration $(\mathrm{nmol} / \mathrm{l})$, $\mathrm{r}^{2}=0.999$. The method linearity up to $600 \mathrm{nmol} / \mathrm{l}$ was acceptable and comparable with a previous method for DBS cortisol [32].

\section{Sensitivity \& measurement range}

The lower limits of measurement were determined by observing the signal to noise $(\mathrm{S} / \mathrm{N})$ ratio of the cortisol concentrations spiked close to expected LLOQ $(9,5$ and $3 \mathrm{nmol} / \mathrm{l}$ ) in blank blood. The $\mathrm{S} / \mathrm{N}$ ratios of DBS samples at $5 \mathrm{nmol} / \mathrm{l}$ were in the range from 10 to 12 , fulfilling the requirement of $S / \mathrm{N}$ ratio $\geq 10$ to qualify as LLOQ. Both 

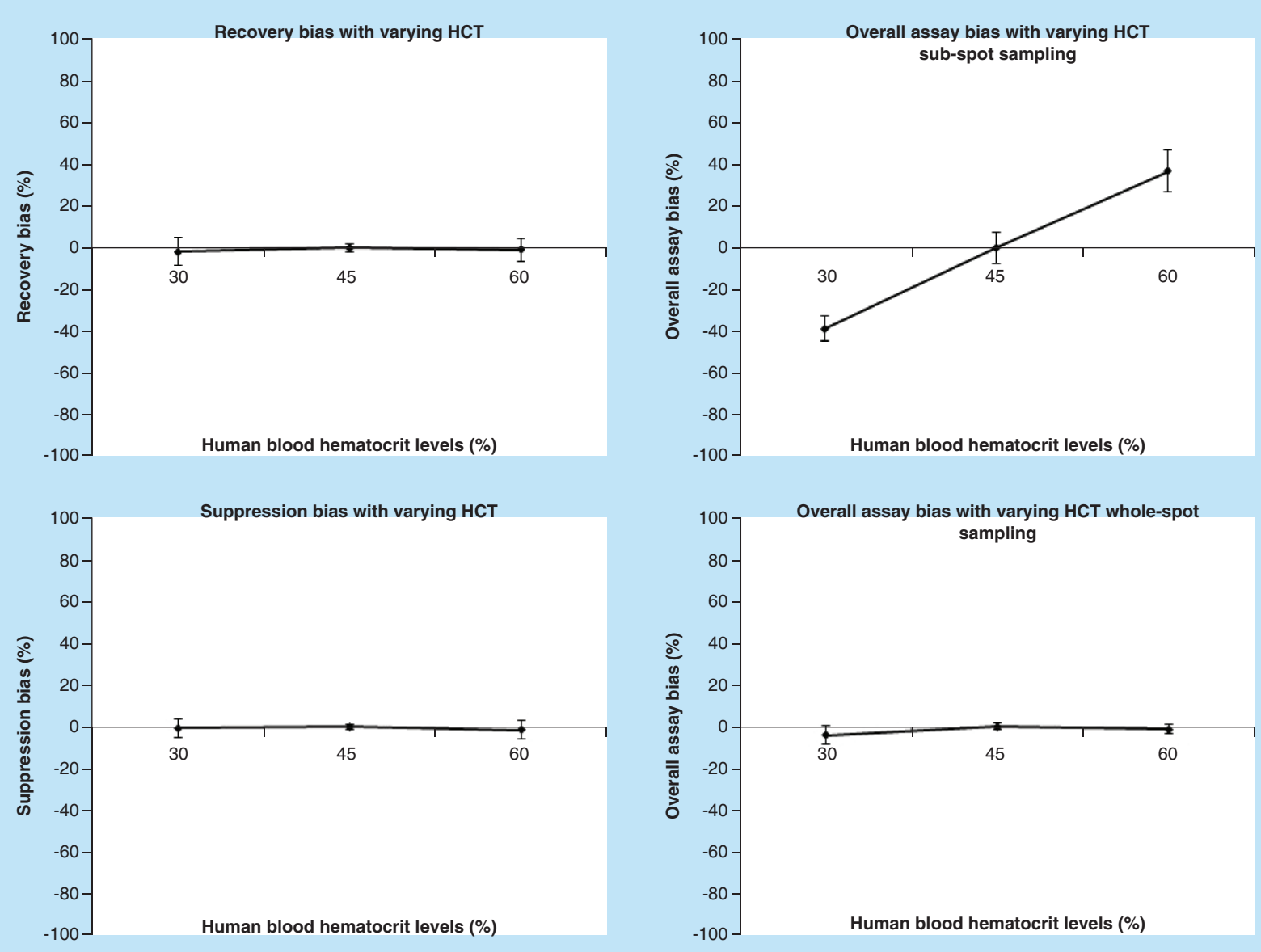

Figure 4. Recovery bias, suppression bias and overall assay bias with varying hematocrit levels. Each data point plotted is a mean bias value $\pm S D(n=6)$ from three repeated experiments $(n=18$ total replicates at each dried blood spot quality control concentration). Bias values at varying hematocrit levels (30 and 60\%) have been calculated relative to the results at mean hematocrit level (45\%).

accuracy and precision were within the pre-defined acceptance limits of $20 \%$, thus $5 \mathrm{nmol} / \mathrm{l}$ was set as the LLOQ of this analytical method, very close to the LLOQ value $(3.4 \mathrm{nmol} / \mathrm{l})$ from another study [32].

Linearity of the method in samples with cortisol above the upper limit of the calibration range $(5-600 \mathrm{nmol} / \mathrm{l})$ was tested with serial dilutions (50/50) of a DBS sample extract with a blank DBS extract. It was found to be linear $\left(\mathrm{r}^{2}=0.999\right)$ up to the measured concentrations of $3500 \mathrm{nmol} / \mathrm{l}$.

\section{Selectivity}

A representative LC-MS/MS chromatogram of blank DBS spiked with cortisol at LLOQ $(5 \mathrm{nmol} / \mathrm{l})$ and other possible interfering isobaric endogenous compounds are shown in Figure 5. Analyses of the DBS samples with and without spikes of other steroids showed no difference in the chromatography pattern, nor in the concentrations measured. There was a baseline resolution of cortisol, and no interfering peaks of other tested steroids were observed at the retention time of cortisol. Thus, the chromatographic method clearly distinguished cortisol from possible endogenous isobaric interferences $(20 \alpha$-dihydrocortisone, $20 \beta$-dihydrocortisone, $5 \beta$-dihydrocortisone and 11-epihydrocortisone).

\section{Carry-over}

Sample carry-over was examined by measuring the response of Fluispotter-generated DBS samples with low and high concentration of cortisol in successive injections. Lower cortisol concentrations in both DBS QC samples and DBS calibrators were analyzed, followed by higher cortisol concentrations and again followed by the same low 


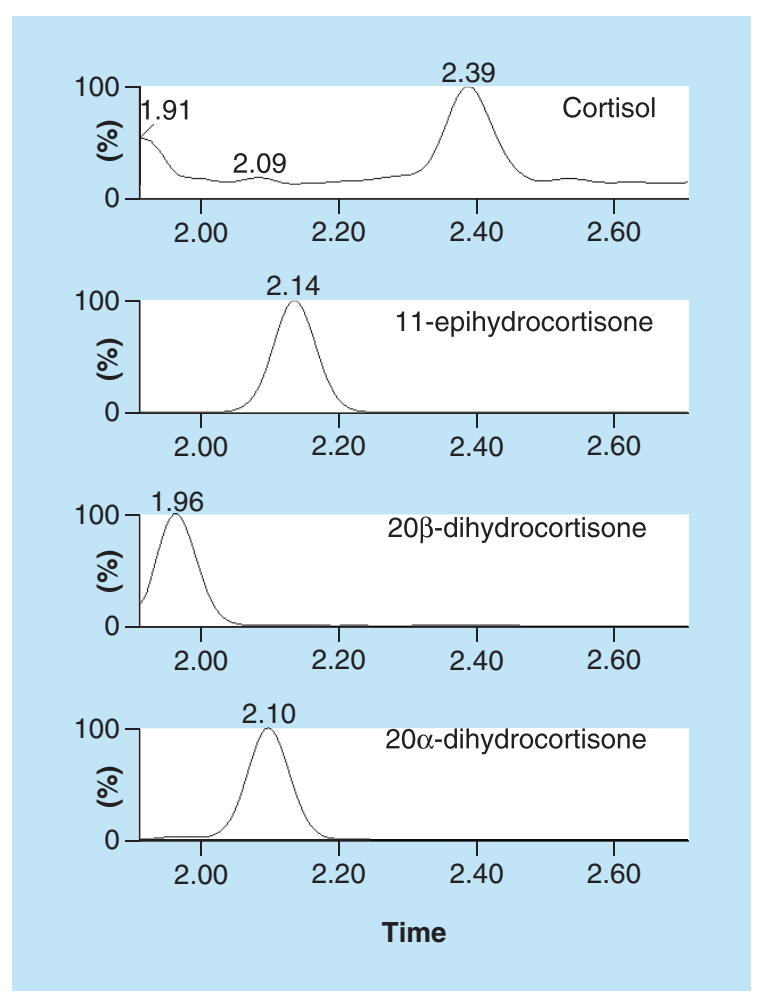

Figure 5. Representative extracted ion chromatograms of a dried blood spot spiked with cortisol (at lower limit of quantification $5 \mathrm{nmol} / \mathrm{l}), 11$-epihydrocortisone, $20 \alpha$-dihydrocortisone and 20ß-dihydrocortisone.

concentration samples, three-times each. The analysis of a DBS sample at upper limit of quantification did not show any signal of carry-over in the succeeding blank solvent. No sample carry-over was found.

\section{Stability}

Long-term stability testing of cortisol in DBS samples was carried out at the selected storage conditions (room temperature, 4 and $-18^{\circ} \mathrm{C}$ ) at different time points over a period of 6 months at two concentrations of cortisol (low: $12 \mathrm{nmol} / \mathrm{l}$ and high: $174 \mathrm{nmol} / \mathrm{l})$. The bias in the cortisol concentrations between stored and fresh DBS samples was between -12 and $5 \%$ for up to 6 months at all three storage temperature conditions (room temperature, 4 and $-18^{\circ} \mathrm{C}$ ) and for up to 12 months at 4 and $-18^{\circ} \mathrm{C}$, demonstrating that cortisol was stable in DBS samples for up to 6 months at room temperature and up to 12 months under refrigeration and freezing. The stability of cortisol for 4 weeks at room temperature and 8 weeks at $4^{\circ} \mathrm{C}$ has been reported previously [33].

\section{Agreement between plasma cortisol, DBS cortisol \& DBS-predicted plasma cortisol concentrations DBS cortisol versus plasma cortisol}

The cortisol concentration range determined in a total of 148 paired human DBS (HCT range 22\% to 55\%) and plasma samples was 5-1110 nmol/l and 7-1646 nmol/l, respectively. Deming regression analysis indicated a relationship (Figure 6) between DBS and plasma-cortisol concentrations $(y=-24.04+0.643 \mathrm{x}, 95 \% \mathrm{CI}$ of slope $\left.=0.59-0.69, r^{2}=952\right)$. The Bland-Altman plot with $95 \%$ limits of agreement (LoA) showed a significant relative difference between DBS-cortisol and plasma-cortisol concentrations of -57\% (95\% LoA -34\% to -80\%) across the measured concentration range.

\section{DBS-predicted plasma cortisol versus measured plasma cortisol}

The median of the DBS/plasma cortisol concentration ratio was 0.54 (range: $0.41-0.78$ ). A linear negative correlation was established between the paired DBS/plasma ratio and individual HCT levels of the 148 human samples (HCT range 22-55\%) using simple linear regression (Figure 7). The negative linear relationship was basically due to the majority of the cortisol distributed in plasma [28] and less plasma volume covered per unit of accurate volume DBS $(10 \mu \mathrm{l})$ with increasing HCT level. The regression equation from this relationship was: $y=-0.876 x+0.920, r^{2}=0.754$ (Figure 7). Plasma cortisol concentrations were thus predicted from DBS cortisol 

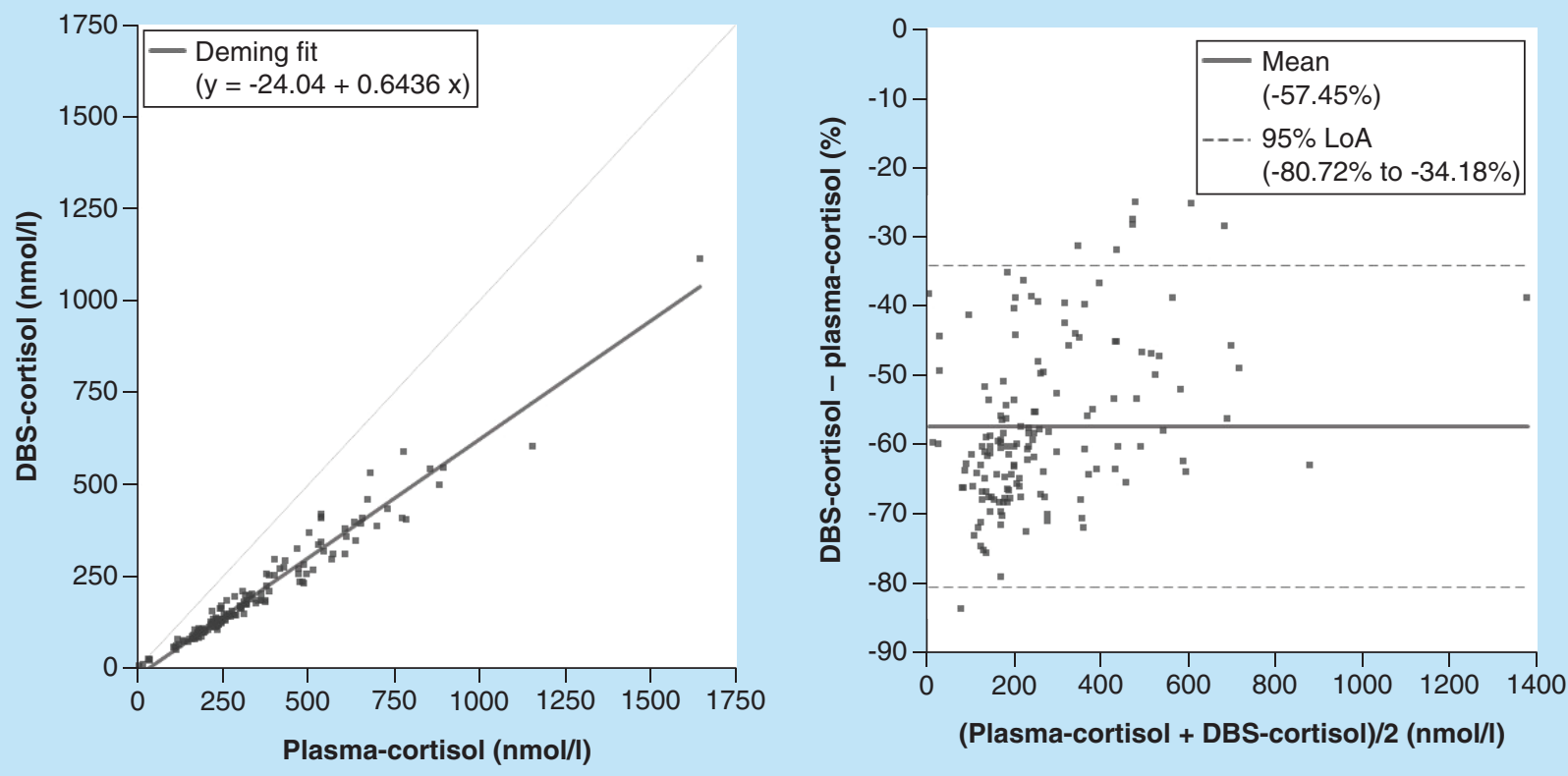

Figure 6. Deming regression and Bland-Altman analyses of cortisol concentrations in dried blood spot plotted against the corresponding plasma conce DBS: Dried blood spot.

concentrations after correction for individual HCT, slope, and intercept factors using the regression equation, as follows:

$$
\mathrm{PC}_{\text {plasma }}=\frac{\mathrm{C}_{\mathrm{DBS}}}{[0.920+(-0.876) \mathrm{HCT}]}
$$

where $P C_{\text {plasma }}$ is the DBS-predicted plasma cortisol concentration, $C_{D B S}$ is the measured DBS-cortisol concentration, -0.876 is the slope of the regression line with an intercept of 0.920 from the regression equation, and HCT is the HCT value for each sample. Others have used other ways of calculating plasma concentrations from DBS concentrations using information from HCT, fraction bound to plasma protein, ratio of blood to serum concentration, red blood cell to plasma distribution, etc. [34].

A linear relationship was established between DBS-predicted plasma-cortisol and measured plasma-cortisol concentrations over the entire concentration range $\left(r^{2}=0.982\right)$. According to Deming regression analysis (Figure 7$)$, a negligible variable proportional bias (slope estimate of $1.09,95 \%$ CI: $1.00-1.19)$ was observed with an intercept -28.86 (95\% CI: -57.13 to -0.58$)$. Bland-Altman analysis showed a mean difference of DBS-predicted and measured plasma-cortisol of $-0.20 \%$ (95\% LoA: -12.12 to $11.72 \%$ ). This indicated that plasma equivalent cortisol concentrations were predicted from the DBS-cortisol concentrations after correction for individual HCT, slope, and intercept factors using the regression equation of the DBS/plasma cortisol ratio plotted against HCT. Plasma equivalent cortisol concentrations were also predicted from the DBS-cortisol concentrations applying a regular conversion method correcting for individual HCT (DBS concentration/1-HCT) as applied in few studies [34]. This method also showed a linear relationship between DBS-predicted and measured plasma-cortisol concentrations with a mean difference for cortisol of $-5.07 \%$ (95\% LoA: -17.17 to $7.02 \%)$.

\section{Conclusion}

In summary, a DBS-LC-MS/MS bioanalytical method was developed for the determination of cortisol in samples automatically spotted by a novel wearable and automated microsampling device, Fluispotter. The automated DBS sampling by Fluispotter was qualified by comparing manual DBS sampling and plasma sampling methods. This is a first report showing high accuracy and precision of cortisol determinations in DBS samples spotted by Fluispotter. The validated DBS-LC-MS/MS quantitative method was linear, selective, sensitive, and independent of HCT 

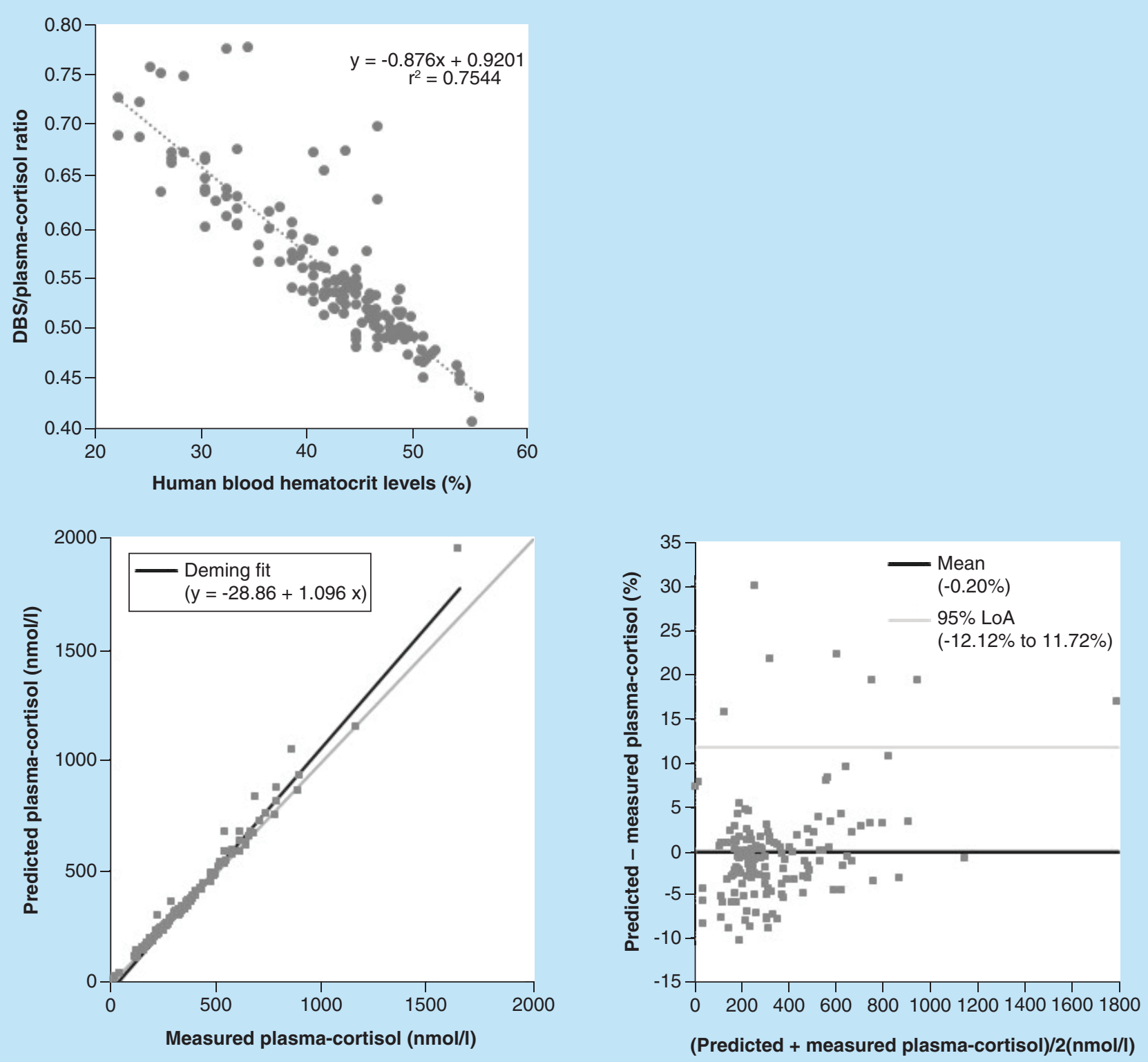

Figure 7. A simple linear regression of dried blood spot/plasma cortisol ratio against the hematocrit level of individual subjects. Deming regression (lower left) and Bland-Altman (lower right) analysis of the DBS-predicted plasma cortisol concentrations plotted against the corresponding measured plasma cortisol concentrations in paired samples $(n=148)$. The Bland-Altman plot shows the mean difference (solid line) and $95 \%$ limits of agreements (dashed lines).

variations. The plasma equivalent cortisol concentrations could be predicted from DBS cortisol measurements after correction for individual HCT. Our experiments also support literature findings that HCT affects the overall assay bias in quantitative bioanalysis upon sub-spot analysis, and this effect can be overcome by analyzing the entire DBS sample spotted by Fluispotter. In conclusion, Fluispotter demonstrated the ability to spot accurate and precise blood volumes in the form of DBS and the potential of this technique in serial DBS sampling in clinical pharmacokinetics studies.

\section{Future perspective}

The development of wearable and automated DBS sampling devices is important for serial blood sampling in clinical pharmacokinetics studies. The benefits as well as the problems associated with the practical field performance of 
the Fluispotter microsampling device will come forward in upcoming clinical applications. Overall, Fluispotter performed well in laboratory conditions within standard method validation acceptance criteria and if it is successful in upcoming clinical application tests, it has great potential in automated serial microsampling in clinical practice, for example, for diagnostic purposes and treatment evaluation - including the pediatric population - as well as in pharmacokinetic studies. Fluispotter needs a connection with venous blood circulation through a catheter, but nevertheless this automated sampling reduces human error in blood spotting, collecting samples without stressing the patient and allowing sampling at sleep and exercise, thereby improving the clinical outcome significantly.

Executive summary

\section{Background}

- Hematocrit (HCT) is a major challenge in dried blood spot (DBS) quantitative bioanalysis in getting data with the same robustness and confidence as with plasma samples.

- A novel automated and accurate volume serial DBS microsampling device, 'Fluispotter' was tested for sampling performance in laboratory conditions and for the ability to overcome the issue associated with blood HCT.

Experimental

- A fixed blood sample volume of $10 \mu \mathrm{l}$ was serially spotted by Fluispotter in a laboratory condition.

- A quantitative DBS-LC-MS/MS assay was developed and validated, using cortisol as a model compound.

- Sampling volume variations were compared between Fluispotter and manual spotting.

- Cortisol concentrations in 148 paired human plasma and DBS samples across the different HCTs range (22-55\%) were compared.

\section{Results \& discussion}

- Fluispotter can deliver 20 serial DBS samples with high accuracy and precision ( $<10 \%$ was determined by LC-MS/MS measurements of cortisol in DBS samples).

- The average blood volume in DBS samples spotted automatically by Fluispotter and manually by $10-\mu \mathrm{l}$ micropipettes differed by $3 \%$ with the same level of precision ( $\% \mathrm{CV},<3 \%$ ).

- Overall assay bias for the test compound, cortisol, was negligible across the HCTs tested (30-60\%) when analyzing the whole-spot DBS samples.

- DBS-predicted plasma cortisol compared with measured plasma cortisol revealed negligible proportional bias (slope 1.096; 95\% Cl: 1.003-1.189) and a constant bias (intercept -28.86; 95\% Cl: -57.13 to -0.588 ).

- Bland-Altman analysis showed a mean percent difference of DBS-predicted plasma to measured plasma cortisol concentrations of $0.20 \%$, (95\% LoA: -12.12 to $11.72 \%$ ).

Conclusion

- The novel automated Fluispotter can spot serial DBS samples with high accuracy and precision.

- Fluispotter has the potential to overcome sample blood volume variations associated with HCT in DBS quantitative bioanalysis.

- Fluispotter showed the potential for serial DBS microsampling in human pharmacokinetics studies.

\section{Acknowledgments}

The authors would like to thank Innovation Fund Denmark for providing a research grant. Many thanks to PerkinElmer Denmark for providing the Wallac DBS Puncher (PerkinElmer, MA, USA).

Financial \& competing interests disclosure

This work was funded by Innovation Fund Denmark (\#5164-00008B). M Rohde, S Velschow are employed by Fluisense ApS, the inventor of Fluispotter. The authors have no other relevant affiliations or financial involvement with any organization or entity with a financial interest in or financial conflict with the subject matter or materials discussed in the manuscript apart from those disclosed.

No writing assistance was utilized in the production of this manuscript.

\section{Open access}

This work is licensed under the Attribution-NonCommercial-NoDerivatives 4.0 Unported License. To view a copy of this license, visit http://creativecommons.org/licenses/by-nc-nd/4.0/ 


\section{References}

Papers of special note have been highlighted as: $\bullet$ of interest; $\bullet \bullet$ of considerable interest

1. Barfield M, Spooner N, Lad R, Parry S, Fowles S. Application of dried blood spots combined with HPLC-MS/MS for the quantification of acetaminophen in toxicokinetic studies. J. Chromatogr. B Analyt. Technol. Biomed. Life Sci. 870(1), 32-37 (2008).

2. Chen G, Jirjees F Sr, Al Bawab A, Mcelnay JC. Quantification of amlodipine in dried blood spot samples by high performance liquid chromatography tandem mass spectrometry. J. Chromatogr. B Analyt. Technol. Biomed. Life Sci. 1072, 252-258 (2018).

3. Choi R, Park HD, Oh HJ, Lee K, Song J, Lee SY. Dried blood spot multiplexed steroid profiling using liquid chromatography tandem mass spectrometry in Korean neonates. Ann. Lab. Med. 39(3), 263-270 (2019).

4. Guthrie R, Susi A. A simple phenylalanine method for detecting phenylketonuria in large populations of newborn infants. Pediatrics 32 , 338-343 (1963).

5. Mei JV, Alexander JR, Adam BW, Hannon WH. Use of filter paper for the collection and analysis of human whole blood specimens. J. Nutr. 131(5), 1631S-1636S (2001).

6. De Vries R, Barfield M, Van De Merbel N et al. The effect of hematocrit on bioanalysis of DBS: results from the EBF DBS-microsampling consortium. Bioanalysis 5(17), 2147-2160 (2013).

7. Denniff P, Spooner N. The effect of hematocrit on assay bias when using DBS samples for the quantitative bioanalysis of drugs. Bioanalysis 2(8), 1385-1395 (2010).

- Shows how hematocrit affects the area bias, thereby affecting the overall assay bias on quantitative DBS bioanalysis.

8. Abu-Rabie P, Denniff P, Spooner N, Chowdhry BZ, Pullen FS. Investigation of different approaches to incorporating internal standard in DBS quantitative bioanalytical workflows and their effect on nullifying hematocrit-based assay bias. Anal. Chem. 87(9), 4996-5003 (2015).

- Great report showing how hematocrit affects the area, recovery and suppression bias, thereby affecting the overall assay bias on quantitative DBS bioanalysis.

9. Denniff P, Spooner N. Volumetric absorptive microsampling: a dried sample collection technique for quantitative bioanalysis. Anal. Chem. 86(16), 8489-8495 (2014).

-. First report of volumetric absorptive microsampling for the collection of accurate blood volumes independent of hematocrit.

10. Leuthold LA, Heudi O, Deglon J et al. New microfluidic-based sampling procedure for overcoming the hematocrit problem associated with dried blood spot analysis. Anal. Chem. 87(4), 2068-2071 (2015).

11. Deprez S, Paniagua-Gonzalez L, Velghe S, Stove CP. Evaluation of the performance and hematocrit independence of the hemapen as a volumetric dried blood spot collection device. Anal. Chem. 91(22), 14467-14475 (2019).

12. Lenk G, Ullah S, Stemme G, Beck O, Roxhed N. Evaluation of a volumetric dried blood spot card using a gravimetric method and a bioanalytical method with capillary blood from 44 volunteers. Anal. Chem. 91(9), 5558-5565 (2019).

13. Neto R, Gooley A, Breadmore MC, Hilder EF, Lapierre F. Precise, accurate and user-independent blood collection system for dried blood spot sample preparation. Anal. Bioanal. Chem. 410(14), 3315-3323 (2018).

14. Spooner N, Denniff P, Michielsen L et al. A device for dried blood microsampling in quantitative bioanalysis: overcoming the issues associated blood hematocrit. Bioanalysis 7(6), 653-659 (2015).

15. Lenk G, Sandkvist S, Pohanka A, Stemme G, Beck O, Roxhed N. A disposable sampling device to collect volume-measured DBS directly from a fingerprick onto DBS paper. Bioanalysis 7(16), 2085-2094 (2015).

16. Teilmann AC, Kalliokoski O, Sorensen DB, Hau J, Abelson KS. Manual versus automated blood sampling: impact of repeated blood sampling on stress parameters and behavior in male NMRI mice. Lab. Anim. 48(4), 278-291 (2014).

17. Newman BH, Pichette S, Pichette D, Dzaka E. Adverse effects in blood donors after whole-blood donation: a study of 1000 blood donors interviewed 3 weeks after whole-blood donation. Transfusion 43(5), 598-603 (2003).

18. Herndon JG, Turner JJ, Perachio AA, Blank MS, Collins DC. Endocrine changes induced by venipuncture in rhesus monkeys. Physiol. Behav. 32(4), 673-676 (1984).

19. Uyeda C, Pham R, Fide $S$ et al. Application of automated dried blood spot sampling and LC-MS/MS for pharmacokinetic studies of AMG 517 in rats. Bioanalysis 3(20), 2349-2356 (2011).

20. Velschow S. Fluisense. A body fluid sampling device. European Union EP 2373221 B1 (2013).

21. Velschow S. Fluisense. Multi lumen catheter. European Union EP 2884895 B1 (2016).

22. Velschow S. Fluisense. Improved fluid sampling system. European Union EP 2884896 B1 (2016).

23. International Council for Harmonisation of Technical Requirements for Pharmaceuticals for Human Use. ICH Harmonized Tripartite Guideline: validation of analytical procedures: text and methodology Q2(R1)(2005).

24. US FDA. Bioanalytical method validation: guidance for industry (2018).

25. Timmerman P, White S, Globig S, Ludtke S, Brunet L, Smeraglia J. EBF recommendation on the validation of bioanalytical methods for dried blood spots. Bioanalysis 3(14), 1567-1575 (2011). 
- Provides standard sets for DBS quantitative bioanalysis.

26. Timmerman P, White S, Cobb Z, De Vries R, Thomas E, Van Baar B. Update of the EBF recommendation for the use of DBS in regulated bioanalysis integrating the conclusions from the EBF DBS-microsampling consortium. Bioanalysis 5(17), 2129-2136 (2013).

27. Spooner N, Lad R, Barfield M. Dried blood spots as a sample collection technique for the determination of pharmacokinetics in clinical studies: considerations for the validation of a quantitative bioanalytical method. Anal. Chem. 81(4), 1557-1563 (2009).

28. Peterson RE, Wyngaarden JB, Guerra SL, Brodie BB, Bunim JJ. The physiological disposition and metabolic fate of hydrocortisone in man. J. Clin. Invest. 34(12), 1779-1794 (1955).

29. Monostori P, Szabo P, Marginean O, Bereczki C, Karg E. Concurrent confirmation and differential diagnosis of congenital adrenal hyperplasia from dried blood spots: application of a second-tier LC-MS/MS assay in a cross-border cooperation for newborn screening. Horm. Res. Paediatr. 84(5), 311-318 (2015).

30. Seo JY, Park HD, Kim JW et al. Steroid profiling for congenital adrenal hyperplasia by tandem mass spectrometry as a second-tier test reduces follow-up burdens in a tertiary care hospital: a retrospective and prospective evaluation. J. Perinat. Med. 42(1), 121-127 (2014).

31. Dhillon K, Ho T, Rich P et al. An automated method on analysis of blood steroids using liquid chromatography tandem mass spectrometry: application to population screening for congenital adrenal hyperplasia in newborns. Clin. Chim. Acta 412(23-24), 2076-2084 (2011).

32. Janzen N, Sander S, Terhardt M et al. Rapid steroid hormone quantification for congenital adrenal hyperplasia (CAH) in dried blood spots using UPLC liquid chromatography-tandem mass spectrometry. Steroids 76(13), 1437-1442 (2011).

33. Worthman CM, Stallings JF. Hormone measures in finger-prick blood spot samples: new field methods for reproductive endocrinology. Am. J. Phys. Anthropol. 104(1), 1-21 (1997).

34. Enderle Y, Foerster K, Burhenne J. Clinical feasibility of dried blood spots: analytics, validation, and applications. J. Pharm. Biomed. Anal. 130, 231-243 (2016). 